\title{
Expression of MMP-2 and TIMP-3 with incidence and prognosis of giant-cell tumor of the bone
}

\author{
ZHENDONG LIU ${ }^{1 *}$, YUXIN REN ${ }^{2 *}$ and FUQIANG ZHU ${ }^{2}$ \\ Departments of ${ }^{1}$ Orthopedics and ${ }^{2}$ Spine Surgery, Dezhou People's Hospital, \\ Dezhou, Shandong 253014, P.R. China
}

Received August 8, 2017; Accepted January 2, 2018

DOI: $10.3892 / \mathrm{ol} .2018 .8696$

\begin{abstract}
The expression of matrix metalloproteinase-2 (MMP-2) and tissue inhibitor of matrix metalloproteinase-3 (TIMP-3) in giant-cell tumor of bone (GCT), and the correlation of their expression with the clinicopathologic features and prognosis were investigated. A total of $70 \mathrm{GCT}$ patients treated in our hospital from September, 2013 to September, 2015, were selected, and the tumor and para-carcinoma tissues were obtained by surgery. The expression levels of MMP-2 and TIMP-3 in GCT and para-carcinoma tissues were detected via semi-quantitative polymerase chain reaction (PCR) and western blot analysis, as well as immunohistochemical staining. Moreover, the clinicopathological data of the GCT patients were collected to study the correlation of MMP-2 and TIMP-3 with clinicopathological features and prognosis of GCT. The results of semi-quantitative PCR and western blot analysis revealed that the expression level of MMP-2 in tissues of the 70 GCT patients was significantly higher than that in para-carcinoma tissues, and the difference was statistically significant $(\mathrm{P}<0.01)$, while the expression level of TIMP-3 was obviously lower than that in para-carcinoma tissues $(\mathrm{P}<0.01)$ The results of immunohistochemical staining revealed that the positive expression rate of MMP-2 was $57.6 \%$ in GCT tissues and $18.9 \%$ in para-carcinoma tissues, while that of TIMP-3 was $63.2 \%$ in GCT tissues and $13.8 \%$ in para-carcinoma tissues, and the differences were statistically significant $(\mathrm{P}<0.01)$. The expression levels of MMP-2 and TIMP-3 were correlated with the diameter of tumor, clinical staging, lymph node metastasis and relapse of GCT $(\mathrm{P}<0.01)$, but were not correlated with the age and sex of GCT patients $(\mathrm{P}>0.05)$. There was a negative correlation between MMP-2 and TIMP-3 expression levels
\end{abstract}

Correspondence to: Dr Fuqiang Zhu, Department of Spine Surgery, Dezhou People's Hospital, 1751 Xinhu Street, Dezhou, Shandong 253014, P.R. China

E-mail:wwwjinan007@163.com

*Contributed equally

Key words: matrix metalloproteinase-2, tissue inhibitor of matrix metalloproteinase-3, giant-cell tumor, prognosis $(\mathrm{r}=-0.258, \mathrm{P}<0.05)$. The expression levels of MMP-2 and TIMP-3 are closely related to the clinicopathological features and prognosis of patients, which can be used as one of the clinical examination indexes of GCT and also provide new insights for the clinical treatment of GCT.

\section{Introduction}

Giant-cell tumor of the bone (GCT) is a common primary bone tumor. According to epidemiological surveys, GCT accounts for $4-5 \%$ of all primary bone tumors and occurs more frequently in women, and the incidence rate is higher in China than that in western countries. The lesion is common in the epiphysis, and the larger lesion can spread to the metaphysis, even the backbone (1-3). With the development of medical technology, the detection and treatment rates of GCT have been significantly increased, but its onset is slow and there are no obvious clinicopathological features in the early stage; thus, although $80 \%$ of GCT tumors are benign, approximately $20 \%$ can still spread to the lung tissues and other parts, causing deterioration. The preferred treatment method of GCT is surgery (4-6). Matrix metalloproteinase (MMP) is a proteolytic enzyme family capable of degrading basement membrane (BM) and extracellular matrix (ECM). MMP-2 is one of the important members in the MMP family and is the main enzyme of degrading type IV collagen. Tumor cells degrade the BM and ECM under the blood capillary endodermis through the secretion of MMPs, making the endothelial cells migrate and proliferate outwarda, ultimately forming new blood vessels and $\operatorname{BM}(7,8)$. Tissue inhibitor of metalloproteinase-3 (TIMP-3) is a widely-distributed endogenous MMP inhibitor and a new member in the TIMP family, as well as an MMP-specific full-function inhibitor, which has a certain inhibitory effect on tumor growth through inhibition of MMPs (9). However, there is no previous report on the correlation of the expression levels of MMP-2 and TIMP-3 with the incidence and prognosis of GCT.

In the present study, the expression levels of MMP-2 and MMP-3 in GCT patients were detected and the correlation of expression levels of MMP-2 and TIMP-3 with clinicopathological features and prognosis of GCT was investigated, to determine the relationship between the occurrence and development of GCT and the expression levels of MMP-2 and TIMP-3. 


\section{Materials and methods}

Objects. The 70 samples in the present study were paraffin samples obtained from the surgical resection of patients treated and diagnosed as GCT via pathological examination in Dezhou Hospital (Shandong, China) from September, 2013 to September, 2015. These patients were aged 38-87 years, including 32 males and 38 females. Other consumptive diseases were eliminated from all the patients enrolled, and patients signed the informed consent. The patients had complete clinical and pathological data. At the same time, the para-carcinoma tissues ( $>3 \mathrm{~cm}$ away from the cancer tissue) of the above patients were collected as the control group. Patients enrolled had a 1 year follow-up record and the complete treatment regimen. The tumor and para-carcinoma tissue samples were stored in liquid nitrogen at at $-196^{\circ} \mathrm{C}$.

Instruments and materials. MMP-2 monoclonal antibody, TIMP-3 and GAPDH monoclonal antibody (cat. nos. 04-1048, AB6000 and MABS819 all from EMD Millipore, Billerica, MA, USA), TRIzol, immunohistochemistry and reverse transcription kit (all from Invitrogen; Thermo Fisher Scientific, Inc., Waltham, MA, USA), inverted fluorescence microscope (Thermo Fisher Scientific, Inc.), sheep anti-rabbit IgG secondary antibody (cat. no. AP510, EMD Millipore, Billerica, MA, USA), pipettor (Eppendorf, Hamburg, Germany), polymerase chain reaction (PCR) instrument (ABI USA, Vernon, CA, USA), and an ultraviolet imaging system (Biometra $\mathrm{GmbH}$, Göttingen, Germany) were used in the present study. The origin and batch number of other relevant instruments and materials are specified in corresponding parts.

Detection of expression levels of MMP-2 and TIMP-3 in cancer tissues of GCT patients via semi-quantitative PCR. The total RNA was extracted from tissues using the TRIzol kit according to the manufacturer's instructions after the tumor and para-carcinoma tissue samples were obtained from GCT patients and thawed, and the RNA integrity was confirmed via agarose gel electrophoresis. The electrophoresis results showed that the $28 \mathrm{~S}, 18 \mathrm{~S}$ and $5 \mathrm{~S}$ bands were clear, and the brightness of the $28 \mathrm{~S}$ band was about twice that of $18 \mathrm{~S}$, indicating that the RNA was intact and could be used for subsequent experiments. After cDNA was obtained via reverse transcription using the reverse transcription kit, the expression of MMP-2 and TIMP-3 in tumor and para-carcinoma tissues were detected via semi-quantitative PCR with $G A P D H$ as the internal reference. The reaction conditions were: $95^{\circ} \mathrm{C}$ for $30 \mathrm{sec}, 64^{\circ} \mathrm{C}$ for $25 \mathrm{sec}$ and $72^{\circ} \mathrm{C}$ for $30 \mathrm{sec}$, a total of 35 cycles. Primers were produced by Tiangen Biotech Co., Ltd. (Beijing, China). The sequences are shown in Table I. After the reaction, agarose gel electrophoresis was performed, followed by observation via the ultraviolet imaging system.

Detection of the expression levels of MPP-2 and TIMP-3 via western blot analysis. The tumor and para-carcinoma tissue samples of GCT patients were removed from the liquid nitrogen and cut into pieces with the scissors. The tissues were homogenized by adding the lysis buffer (1:20). After centrifugation at $8,000 \mathrm{xg}$ for $15 \mathrm{~min}$, the supernatant was completely transferred and the total protein was obtained. The
Table I. PCR primers.

\begin{tabular}{ll}
\hline Gene name & \multicolumn{1}{c}{ Sequence } \\
\hline$M M P-2$ & F: 5'-ATCCACCTTGACGATGCTTTAC-3' \\
& R: 5'-TTCAGATGTTCTAAGCCTACGG-3' \\
$T I M P-3$ & F: 5'-TGGCCCTCGTAGCCTTGAGGAC-3' \\
& R: 5'-CCAGTGCTGCAGGGTCCGAGGT-3' \\
GAPDH & F: 5'-GATGATTGGCATGGCTTT-3' \\
& R: 5'-CACCTTCCGTTCCAGTTT-3'
\end{tabular}

MMP-2, matrix metalloproteinase-2; TIMP-3, tissue inhibitor of matrix metalloproteinase-3; GAPDH, glyceraldehyde 3-phosphate dehydrogenase; F, forward; R, reverse.

protein loading sample was then prepared into the loading system at the same concentration via protein quantification. After SDS-PAGE and membrane transfer, the protein was washed and sealed, and the target band was cut. Then rabbit anti-human MPP-2, TIMP-3, GAPDH monoclonal primary antibodies (1:500) was incubated overnight at $4^{\circ} \mathrm{C}$ and washed with Tris-buffered saline with Tween 20 (TBST) three times, and then the secondary antibody $(1: 1,000)$ was incubated at room temperature for $2 \mathrm{~h}$. After being washed with TBST three times, the target protein band was obtained via color development, the exposure was scanned and the results were analyzed with GAPDH as the internal reference. The expression levels of MMP-2 and TIMP-3 in GCT were detected.

Detection of expression levels of MMP-2 and TIMP-3 via immunohistochemistry. The paraffin sections of tumor tissue and para-carcinoma tissue samples of GCT patients were soaked in absolute ethyl alcohol, 95\% ethanol, $75 \%$ ethanol and distilled water for $10 \mathrm{~min}$, respectively, washed with phosphate-buffered saline (PBS) twice (5 min/time), soaked in $3 \% \mathrm{H}_{2} \mathrm{O}_{2}$ at room temperature for $10 \mathrm{~min}$ and then washed with PBS for $5 \mathrm{~min}$. The blocking solution was added dropwise to seal the section for $30 \mathrm{~min}$, and the excess solution was shaken off before MPP-2 and TIMP-3 primary antibodies were added for incubation at $4^{\circ} \mathrm{C}$ overnight. Then the section was washed with PBS three times (5 min/time), and the secondary antibody was added for incubation at room temperature for $1 \mathrm{~h}$. After the section was washed with PBS three times, the horseradish peroxidase was added for labeling for $30 \mathrm{~min}$, followed by color development via diaminobenzidine (DAB) for $5 \mathrm{~min}$, and the section was monitored under the microscope. After the reaction was terminated using distilled water for $30 \mathrm{~min}$, hematoxylin was added for re-staining for 6-9 sec, followed by dehydration, transparency, sealing and microscopic examination. Yellow staining in cytoplasm and surrounding mesenchyme indicated a positive expression of MMP-2, while brown yellow staining in nucleus indicated a positive expression of TIMP-3.

Follow-up. The patients were followed-up during hospitalization and by telephone according to their medical records. The disease progression and survival time of patients were recorded. The patients were followed-up for 1 year, and 
A

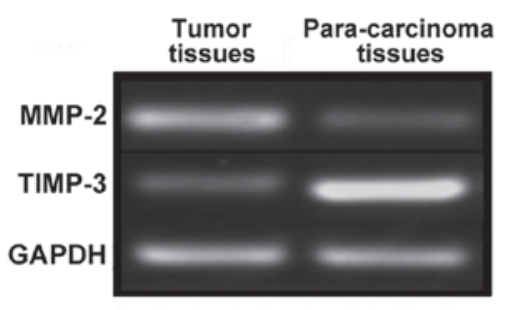

B

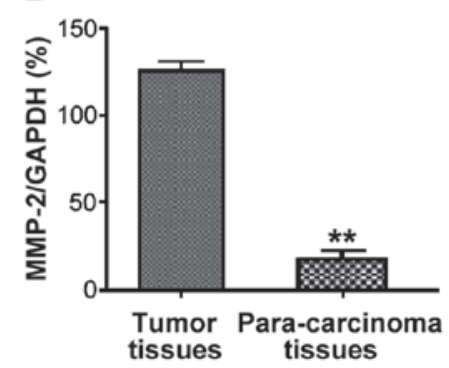

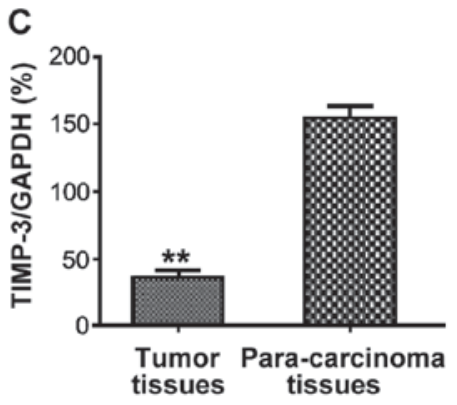

Figure 1. Detection of the relative expression levels of MMP-2 and TIMP-3 in tumor tissues and para-carcinoma tissues of GCT via semi-quantitative PCR. (A) Agarose gel electrophoretogram. (B and C) Statistical charts of the relative expression levels of MMP-2 and TIMP-3: The relative expression level of MMP-2 in tumor tissues of GCT patients is significantly higher than that in para-carcinoma tissues $\left({ }^{* *} \mathrm{P}<0.01\right)$, while the relative expression level of TIMP-3 in tumor tissues of GCT patients is significantly lower than that in para-carcinoma tissues $\left({ }^{* *} \mathrm{P}<0.01\right)$. MMP-2, matrix metalloproteinase-2; TIMP-3, tissue inhibitor of matrix metalloproteinase-3; GAPDH, glyceraldehyde 3-phosphate dehydrogenase.
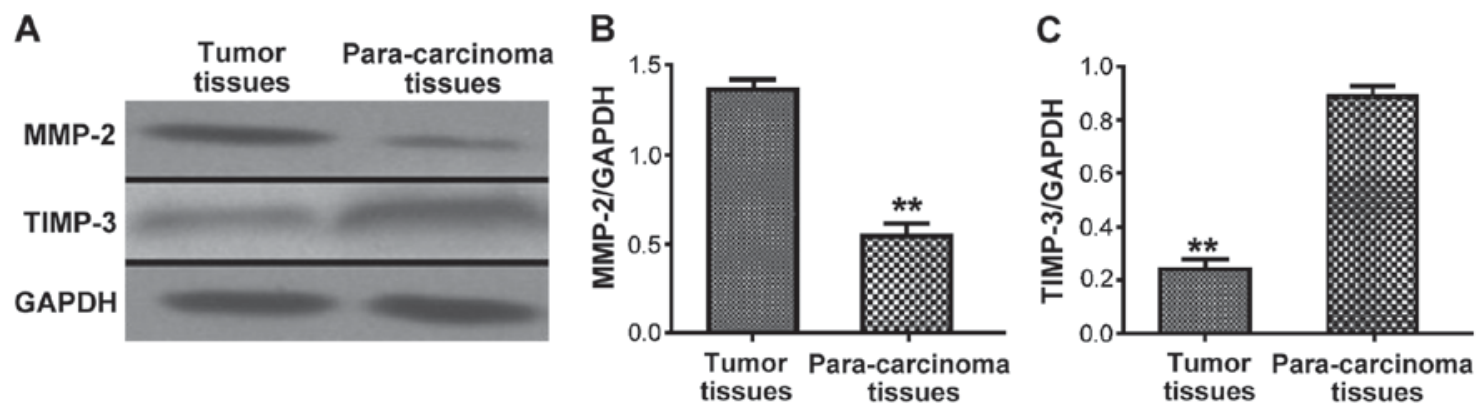

Figure 2. Detection of the protein expression levels of MMP-2 and TIMP-3 in tumor tissues and para-carcinoma tissues of GCT via western blot analysis. (A) Western blot analysis. (B and C) Statistical charts of the expression levels of MMP-2 and TIMP-3: The protein expression level of MMP-2 is significantly higher than that in para-carcinoma tissues $\left({ }^{* *} \mathrm{P}<0.01\right)$, while the protein expression level of TIMP-3 is significantly lower than that in para-carcinoma tissues, and the difference is statistically significant $\left({ }^{* *} \mathrm{P}<0.01\right)$. MMP-2, matrix metalloproteinase-2; TIMP-3, tissue inhibitor of matrix metalloproteinase-3; GAPDH, glyceraldehyde 3-phosphate dehydrogenase.

the detailed follow-up records, examination and hospitalization records were kept.

Statistical analysis. Data in this study are presented as mean \pm standard deviation. SPSS 19.0 software (SPSS, Inc., Chicago, IL, USA) was used for data processing. The t-test was used for measurement data, the Chi-square test was used for the intergroup analysis of enumeration data, and one-way analysis of variance (ANOVA) was used for other data. According to the homogeneity test of variance, if the variance was homogeneous, the Bonferroni method was used for pairwise comparison; otherwise, the Welch method was used for analysis. Dunnett's T3 method was used for multiple comparisons.

\section{Results}

Expression of MMP-2 and TIMP-3 in GCT. A total of 70 tumor and 70 para-carcinoma tissue samples were taken from GCT patients, and the expression levels of MMP-2 and TIMP-3 were detected via semi-quantitative PCR. The results showed that the relative expression level of MMP-2 in tumor tissues in GCT patients was significantly higher than that in para-carcinoma tissues $(\mathrm{P}<0.01)$, while the relative expression level of TIMP-3 was significantly lower than that in para-cancerous tissues, and the difference was statistically significant $(\mathrm{P}<0.01)$ (Fig. 1).
Detection of protein expression of MMP-2 and TIMP-3 in GCT by western blot analysis. The protein expression levels of MMP-2 and TIMP-3 in tumor and para-carcinoma tissue samples of GCT patients were detected via western blot analysis. The results showed that the protein expression level of MMP-2 in tumor tissues in GCT patients was obviously higher than that in para-carcinoma tissues $(\mathrm{P}<0.01)$, while the protein expression level of TIMP-3 was obviously lower than that in para-cancerous tissues, and the difference was statistically significant $(\mathrm{P}<0.01)$ (Fig. 2).

Correlation of MMP-2 and TIMP-3 expression with clinicopathological features and prognosis of patients. The relative expression levels of MMP-2 and TIMP-3 in tumor tissue and para-carcinoma tissue samples of GCT patients were detected by semi-quantitative PCR, and the clinical features of GCT patients, such as age, tumor size and clinical staging, were analyzed. The results revealed that the relative expression levels of MMP-2 and TIMP-3 in the tumor tissues of GCT patients were not correlated with the age and sex of patients $(\mathrm{P}>0.05)$, but closely related to the tumor diameter, clinical staging, lymphatic metastasis and recurrence $(\mathrm{P}<0.05)$ (Table II). The follow-up data of the above patients were divided into high-expression group and normal- or low-expression group according to the relative expression levels of MMP-2 and TIMP-3. The patients were followed-up for 1 year, and the survival time of each group was statistically analyzed. 
Table II. Correlation of relative expression levels of MMP-2 and TIMP-3 with clinical features of patients.

\begin{tabular}{|c|c|c|c|c|c|}
\hline Type & $\mathrm{n}$ & MMP-2 expression level & P-value & TIMP-3 expression level & P-value \\
\hline Age (years) & & & & & 0.0782 \\
\hline$\leq 60$ & 34 & $3.98 \pm 1.33$ & 0.0692 & $5.53 \pm 1.08$ & \\
\hline$>60$ & 36 & $4.16 \pm 1.28$ & & $5.85 \pm 1.12$ & \\
\hline Sex & & & & & 0.0873 \\
\hline Male & 33 & $4.07 \pm 1.08$ & 0.0619 & $4.63 \pm 1.62$ & \\
\hline Female & 37 & $3.96 \pm 1.22$ & & $4.57 \pm 1.29$ & \\
\hline Tumor size & & & & & 0.0098 \\
\hline$<5 \mathrm{~cm}$ & 28 & $2.29 \pm 1.39$ & 0.0072 & $6.08 \pm 1.19$ & \\
\hline$\geq 5 \mathrm{~cm}$ & 42 & $6.89 \pm 1.87$ & & $3.95 \pm 2.01$ & \\
\hline Lymphatic metastasis & & & & & 0.0097 \\
\hline Yes & 46 & $6.76 \pm 2.01$ & 0.0078 & $2.17 \pm 1.78$ & \\
\hline No & 24 & $3.58 \pm 1.96$ & & $6.53 \pm 1.05$ & \\
\hline Clinical staging & & & & & 0.0097 \\
\hline Limited stage disease & 53 & $2.76 \pm 0.83$ & 0.0086 & $5.92 \pm 1.25$ & \\
\hline Extensive stage disease & 17 & $5.37 \pm 1.06$ & & $2.57 \pm 1.03$ & \\
\hline Relapse & & & & & 0.019 \\
\hline Yes & 49 & $5.68 \pm 1.86$ & 0.026 & $3.87 \pm 1.37$ & \\
\hline No & 21 & $4.52 \pm 1.36$ & & $5.96 \pm 1.69$ & \\
\hline
\end{tabular}

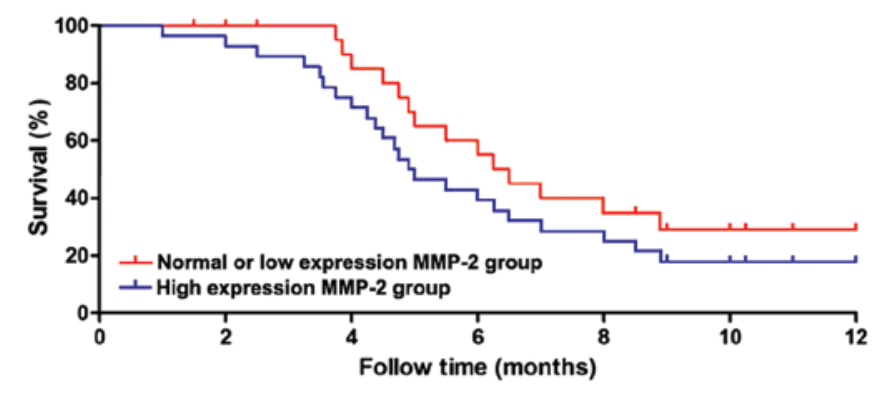

Figure 3. Correlation between MMP-2 expression level and survival time of patients. The survival time of patients in the high-expression MMP-2 group is significantly shorter than that in the normal- or low-expression MMP-2 group, and the difference is statistically significant $(\mathrm{P}<0.01)$. MMP-2, matrix metalloproteinase-2.

The results showed that the survival time of patients in the high-expression MMP-2 group was significantly shorter than that in the normal- or low-expression group $(\mathrm{P}<0.01)$, and the survival curve is shown in Fig. 3. The survival time of patients in the high-expression TIMP-3 group was significantly longer than that in the normal- or low-expression group $(\mathrm{P}<0.01)$, and the survival curve is shown in Fig. 4.

Detection of MMP-2 and TIMP-3 expressions in GCT by immunohistochemistry. The expression levels of MMP-2 and TIMP-3 in GCT were detected by immunohistochemistry. Yellow staining in cytoplasm and surrounding mesenchyme indicated a positive expression of MMP-2, while brown yellow staining in nucleus indicated a positive expression of TIMP-3. The immunohistochemical results of MMP-2 in GCT tissues and para-carcinoma tissues showed that the positive rate of

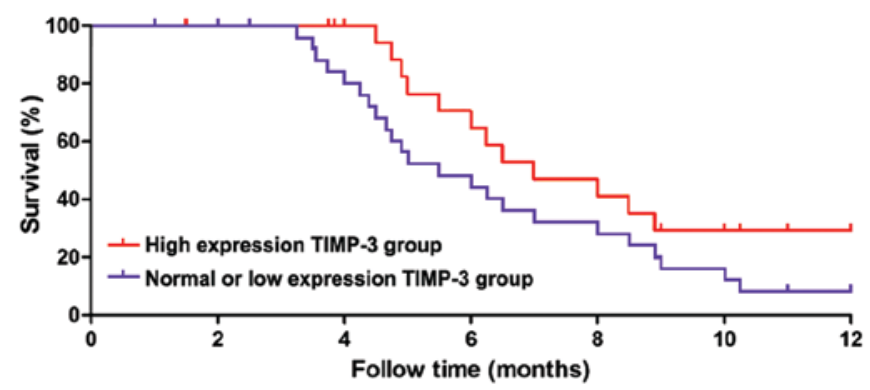

Figure 4. Correlation between TIMP-3 expression level and survival time of patients. The survival time of patients in the high-expression TIMP-3 group is significantly longer than that in the normal- or low-expression TIMP-3 group, and the difference is statistically significant $(\mathrm{P}<0.01)$. TIMP-3, tissue inhibitor of matrix metalloproteinase-3.

MMP-2 in GCT tissues was obviously higher than that in para-carcinoma tissues, and the difference was statistically significant $(\mathrm{P}<0.01)$ (Fig. 5). The immunohistochemical results of TIMP-3 in GCT tissues and para-carcinoma tissues revealed that the positive rate of TIMP-3 in GCT tissues was obviously lower than that in para-carcinoma tissues, and the difference was statistically significant $(\mathrm{P}<0.01)$ (Fig. 6).

Correlation between MMP-2 and TIMP-3 expression in GCT. The expression levels of MMP-2 and TIMP-3 in GCT patients were statistically analyzed. The results showed that the expression level of TIMP-3 in GCT tissues of patients with high MMP-2 expression was lower $(\mathrm{P}<0.05)$. The correlation between MMP-2 and TIMP-3 expression levels revealed that there was a negative correlation between MMP-2 and TIMP-3 expression levels $(\mathrm{r}=-0.258, \mathrm{P}<0.05)$ (Table III). 
A

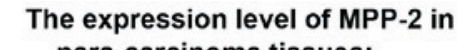

para-carcinoma tissues;

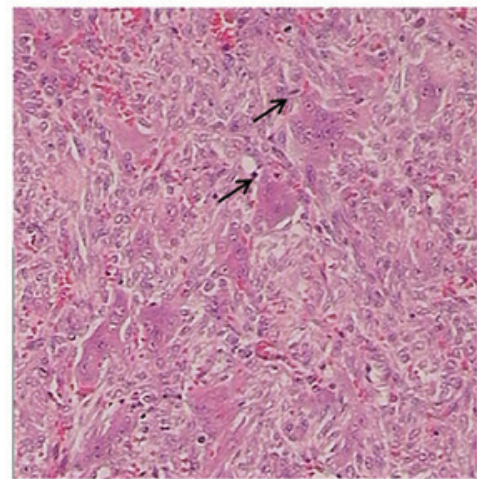

B

The expression level of MPP-2 in GCT

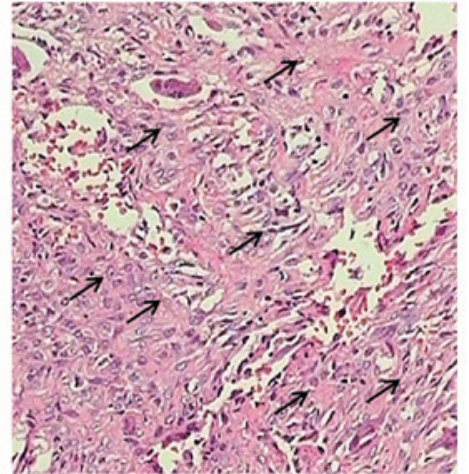

Figure 5. Detection of MPP-2 expression by immunohistochemistry. (A) The expression level of MPP-2 in para-carcinoma tissues. (B) The expression level of MPP-2 in GCT; the results show that the positive expression rate of MPP-2 in GCT is significantly higher than that in para-carcinoma tissues. MMP-2, matrix metalloproteinase-2; GCT giant-cell tumor of bone.

A

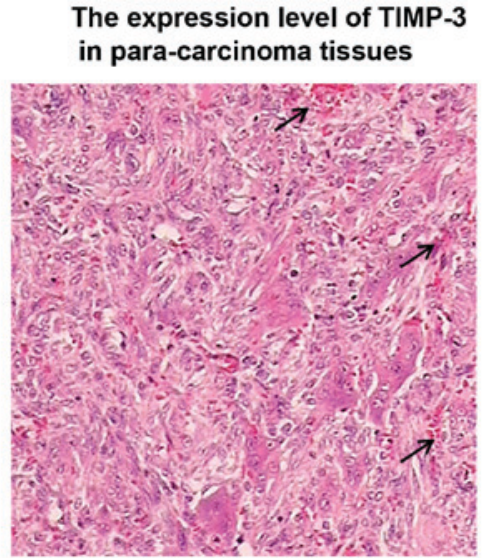

B The expression level of TIMP-3 in GCT

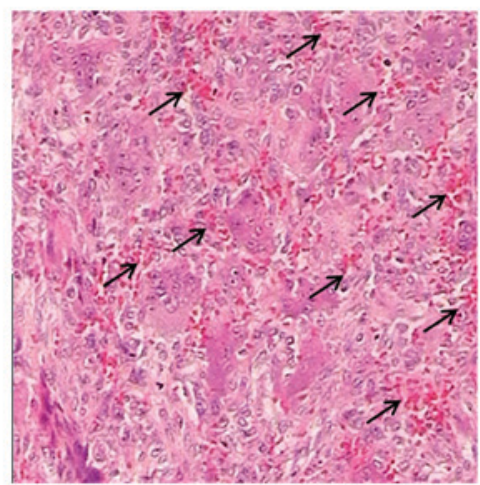

Figure 6. Detection of TIMP-3 expression by immunohistochemistry. (A) The expression level of TIMP-3 in GCT. (B) The expression level of TIMP-3 in para-carcinoma tissues; the results show that the positive expression rate of TIMP-3 in GCT is significantly lower than that in para-carcinoma tissues. TIMP-3, tissue inhibitor of matrix metalloproteinase-3; GCT giant-cell tumor of the bone.

Table III. Correlation of expression of MMP-2 and TIMP-3 in GCT.

\section{TIMP-3}

MMP-2

High Normal or low

expression expression $\quad r \quad P$-value

\begin{tabular}{lcccc}
\hline $\begin{array}{l}\text { High } \\
\text { expression }\end{array}$ & 12 & 58 & & \\
$\begin{array}{l}\text { Normal or } \\
\text { low expression }\end{array}$ & 49 & 21 & -0.258 & 0.021 \\
\hline
\end{tabular}

MMP-2, matrix metalloproteinase-2; TIMP-3, tissue inhibitor of matrix metalloproteinase-3.

\section{Discussion}

GCT, also known as osteoclastoma, is a common and potentially malignant tumor in the bone, characterized by infiltrative growth, easy recurrence and the risk of metastasis to lung tissues $(10,11)$. There are rich blood vessels, including capillary-like blood vessels, in GCT. Previous findings have shown that both microvessel density and VEGF expression level in GCT are very high; in other words, the occurrence and recurrence of GCT are closely related to tumor angiogenesis (12). GCT is often in a more advanced stage when found clinically. Different from other epithelial cell tumors, pathological cells of GCT are difficult to identify in the bone marrow. It has been shown that the incidence of GCT may be closely related to the induction of chromosomal aberrations (13).

MMP is a kind of calcium and zinc atom-dependent protease family, which can degrade almost all the components of ECM. In previous years, it has been shown that the changes and degradation of ECM and BM components are one of the important factors of tumor invasion, metastasis and recurrence (14). TIMP-3, as a widely-distributed endogenous MMP inhibitor, and a non-soluble protein, can closely bind with the ECM components and competitively bind with MMP receptor. Thus, it is a fully functioning MMP inhibitor that can inhibit MMPs and matrix lysin and block the activity of all activated MMPs $(15,16)$. Moreover, TIMP-3 inhibits the formation of tumor vessel by inhibiting the response of 
microvascular endothelial cells to growth factors, and inhibits the tumor progression by regulating cell proliferation and promoting cell apoptosis (17). The analysis of correlation of MMP-2 and TIMP-3 expression with clinicopathological data can effectively explain the pathogenesis of related diseases. Previous findings have shown that MMP-2 can affect tumor angiogenesis, suggesting that MMP-2 is associated with the occurrence and development of a variety of tumors $(18,19)$. Liu et al (20) found that MMP-2 is highly expressed in a variety of solid tumors, indicating that MMP-2 is closely related to the occurrence and metastasis of solid tumors. In the present study, semi-quantitative PCR, western blot analysis and immunohistochemistry were performed to study the expression level of MMP-2 in GCT tissues. The results showed that MMP-2 was highly expressed in GCT at the gene and protein levels, which was closely related to the tumor size, clinical staging, metastasis, recurrence, and the results were also consistent with the report on pulmonary metastasis of GCT. The survival curve of GCT patients during the 1 year follow-up also revealed that the survival time of patients with a high MMP-2 expression was significantly shorter than that with normal or low expression, and the difference was statistically significant $(\mathrm{P}<0.01)$. The above results suggested that MMP-2 is involved in the occurrence and development of GCT, which may lead to metastasis and recurrence of GCT possibly by degrading the BM and ECM. In the present study, it was found that the expression level of TIMP-3 in GCT was significantly lower than that in para-carcinoma tissues, which was closely related to the clinicopathological features, such as the diameter, metastasis and recurrence of GCT. The immunohistochemical results also showed that the expression level of TIMP-3 in para-carcinoma tissues was obviously higher than that in GCT $(\mathrm{P}<0.01)$. The study on the correlation between MMP-2 and TIMP-3 expression levels showed that there was a negative correlation between them. The above results indicated that TIMP-3 may inhibit angiogenesis in GCT and inhibit the activity of MMP-2, thus inhibiting the GCT.

In conclusion, both MMP-2 and TIMP-3 are closely related to the occurrence and development of GCT, which can be used as one of the indexes in the clinical examination of GCT. However, there were still some shortcomings in this study. The pathogenesis was not studied in depth, the sample size in the experiments was small, there were no healthy volunteers for the comparative study, and the results remain to be further confirmed. However, the research value of MMP-2 and TIMP-3 in GCT is unquestionable, which can bring new breakthroughs to the clinical treatment of GCT.

\section{Competing interests}

The authors declare that they have no competing interests.

\section{References}

1. Nicoli TK, Saat R, Kontio R, Piippo A, Tarkkanen M, Tarkkanen J and Jero J: Multidisciplinary approach to management of temporal bone giant cell tumor. J Neurol Surg Rep 77: 144-149, 2016.

2. Hu P, Zhao L, Zhang H, Yu X, Wang Z, Ye Z, Wu S, Guo S, Zhang G, Wang J, et al: Recurrence rates and risk factors for primary giant cell tumors around the knee: A multicentre retrospective study in China. Sci Rep 6: 36332, 2016.
3. Gong HS, Liu GJ, Huang QS, Chen FH and Zhao HB: Surgical management of sacrococcygeal region giant tumors by use of balloon occlusion abdominal aorta. Turk Neurosurg 26: 904-911, 2016.

4. Dabak N,GöçerH and Çıraklı A: Advantages of pressurized-spray cryosurgery in giant cell tumors of the bone. Balkan Med J 33: 496-503, 2016.

5. Rekhi B, Verma V, Gulia A, Jambhekar NA, Desai S, Juvekar SL, Bajpai J and Puri A: Clinicopathological features of a series of 27 cases of post-denosumab treated giant cell tumors of bones: A single institutional experience at a tertiary cancer referral centre, India. Pathol Oncol Res 23: 157-164, 2017.

6. Parra-Herran C1, Schoolmeester JK, Yuan L, Dal Cin P, Fletcher CD, Quade BJ and Nucci MR: Myxoid leiomyosarcoma of the uterus: A clinicopathologic analysis of 30 cases and review of the literature with reappraisal of its distinction from other uterine myxoid mesenchymal neoplasms. Am J Surg Pathol 40: 285-301, 2016.

7. Yang L, Zheng Z, Zhou Q, Bai X, Fan L, Yang C, Su L and Hu D: miR-155 promotes cutaneous wound healing through enhanced keratinocytes migration by MMP-2. J Mol Histol 48: 147-155, 2017.

8. Adhikari N, Mukherjee A, Saha A and Jha T: Arylsulfonamides and selectivity of matrix metalloproteinase-2: An overview. Eur J Med Chem 129: 72-109, 2017.

9. Rother S, Samsonov SA, Moeller S, Schnabelrauch M, Rademann J, Blaszkiewicz J, Köhling S, Waltenberger J, Pisabarro MT, Scharnweber D and Hintze V: Sulfate hyaluronan alters endothelial cell activation in vitro by controlling the biological activity of the angiogenic factors vascular endothelial growth factor-A and tissue inhibitor of metalloproteinase-3. ACS Appl Mater Interfaces 9: 9539-9550, 2017.

10. Shooshtarizadeh T, Rahimi M and Movahedinia S: P63 expression as a biomarker discriminating giant cell tumor of bone from other giant cell-rich bone lesions. Pathol Res Pract 212: 876-879, 2016.

11. Qi DW, Wang P, Ye ZM, Yu XC, Hu YC, Zhang GC, Yan XB, Zheng K, Zhao LM and Zhang HL: Clinical and radiographic results of reconstruction with fibular autograft for distal radius Giant Cell Tumor. Orthop Surg 8: 196-204, 2016.

12. Hu Y, Zhao L, Zhang H, Yu X, Wang Z, Ye Z, Wu S, Guo S, Zhang G, Wang J and Ning X: Sex differences in the recurrence rate and risk factors for primary giant cell tumors around the knee in China. Sci Rep 6: 28173, 2016.

13. Pujani M, Bahadur S, Jairajpuri ZS, Jetley S and Jameel J: Giant cell tumor bone in an elderly male - an unusual case misdiagnosed on MRI as a malignant sarcoma. Indian J Surg Oncol 6: 285-287, 2015

14. Almalki SG, Llamas Valle Y and Agrawal DK: MMP-2 and MMP-14 silencing inhibits VEGFR2 cleavage and induces the differentiation of porcine adipose-derived mesenchymal stem cells to endothelial cells. Stem Cells Transl Med 6: 1385-1398, 2017

15. Yadav PK, Yadav BS, Panigrahi PN, Tripathi V, Chaturvedi N and Kataria M: Molecular characterization and in-silico analysis of the tissue inhibitor of metalloproteinases-3 (TIMP-3) gene of canine mammary tumor. Comb Chem High Throughput Screen 60: 686-691, 2017.

16. Su CW, Su BF, Chiang WL, Yang SF, Chen MK and Lin CW: Plasma levels of the tissue inhibitor matrix metalloproteinase-3 as a potential biomarker in oral cancer progression. Int J Med Sci 14: 37-44, 2017.

17. Sharma K, Tyagi R, Singh R, Sharma SK and Anand A: Serum levels of TIMP-3, LIPC, IER3 and SLC16A8 in CFH negative AMD cases. J Cell Biochem 118: 2087-2095, 2017.

18. Lin HF, Hsi E, Huang LC, Liao YC, Juo SH and Lin RT: Methylation in the matrix metalloproteinase-2 gene is associated with cerebral ischemic stroke. J Investig Med 65: 794-799, 2017.

19. Pillai SS, Yukawa H, Onoshima D, Biju V and Baba Y: Förster resonance energy transfer mediated photoluminescence quenching in stoichiometrically assembled $\mathrm{CdSe} / \mathrm{ZnS}$ quantum dot-peptide labeled black hole quencher conjugates for matrix metalloproteinase-2 sensing. Anal Sci 33: 137-142, 2017.

20. Liu D, Zhang R, Wu J, Pu Y, Yin X, Cheng Y, Wu J, Feng C, Luo $\mathrm{Y}$ and Zhang J: Interleukin-17A promotes esophageal adenocarcinoma cell invasiveness through ROS-dependent, NF-кB-mediated MMP-2/9 activation. Oncol Rep 37: 1779-1785, 2017. International (CC BY-NC-ND 4.0) License. 